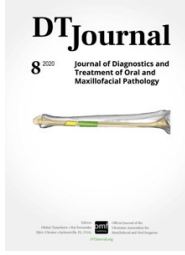

\title{
Surgical Technique for Conservative Management of Benign Lesions in Maxillofacial Region: Experience from a Single Clinic in Bogotá, Colombia
}

\author{
Henry Aldana a, ${ }^{a}$, Sandra Mejía ${ }^{b}, \&$ Camilo Mosquerac
}

\section{SUMMARY}

Odontogenic tumors are lesions derived from the epithelium, the ectomesenchyme and/or the mesenchymal elements that were or continue to be part of the structures that forms the teeth. They are found exclusively within the maxillofacial skeleton and can occur at any stage of an individual's life. Keratocystic odontogenic tumors owe their name to their neoplastic behavior, aggressiveness, and high recurrence rate, which rates from 25 to $65 \%$. Ameloblastomas, on the other hand, are benign, locally aggressive lesions, polymorphic neoplasms with proliferation of the odontogenic epithelium covering a fibrous stroma. Ameloblastomas have a high recurrence rate, about $60-80 \%$ if they are not adequately removed. The management of these injuries has become a challenge for the maxillofacial surgeon, currently there are two treatment alternatives, conservative management and surgical resection. A retrospective review of 7 cases of patients who consulted the maxillofacial surgery department of Clinica Universitaria Colombia was performed. 5 patients with a diagnosis of odontogenic keratocyst and 2 with a diagnosis of ameloblastoma treated with the technique proposed by Dr. Henry Aldana called "enucleation and corticotomies with a rotary instrument." Technique that avoids block resection and allows taking advantage of the residual cavitation defect that, together with the mucoperiosteal closure, contains the initial clot to favor bone neoformation and allows spontaneous regeneration without the need for grafts or any other reconstructive technique. Patients have been followed up for a minimum period of 6 months and bone neoformation has been observed and the patients have no signs or symptoms related to recurrence of the lesion.

\footnotetext{
Bogotá D.C., Colombia

a DDS, Oral and Maxillofacial Surgeon, National University of Colombia, Department of Oral and Maxillofacial surgery, Clinica Universitaria Colombia, Sanitas International Organization.

${ }^{\mathrm{b}}$ DDS, Oral and Maxillofacial Surgeon, National University of Colombia, Private practice.

${ }^{\circ}$ DDS, Oral and Maxillofacial Surgeon, Head of Oral and Maxillofacial Surgery Department, Clinica Santa Maria del Lago, OMFS Clinica Universitaria Colombia, Sanitas International Organization.

* Corresponding author: Clinica Universitaria Colombia, Consultorio 1221, Calle 23 \# 66-46, Bogotá D.C. 111321, Colombia. Tel.: +571 2221479. E-mail: henryaldanacx@hotmail.com (Henry Aldana) Instagram: @maxilogroup
}

Please cite article as: Aldana H, Mejía S, Mosquera C. Surgical technique for conservative Management of benign lesions in Maxillofacial region: Experience from a single clinic in Bogotá, Colombia. J Diagn Treat Oral Maxillofac Pathol 2020;4(8):129-49

Paper received 27 March 2020

Accepted 6 May 2020

Available online 31 August 2020

httpi://dx.doi.org/10.23999/j.dtomp.2020.8.2

C 2020 OMF Publishing, LLC. This is an open access article under the CC BY license (http://creativecommons.org/licenses/by-nc/4.0/). 


\section{INTRODUCTION}

Cons onservative management of benign maxillary lesions has been underestimated. The literature is not benevolent with this management plan.

The proposed technique has shown to have a high success rate, although it does not eliminate the risk of recurrence, it has a considerable impact on the patient by reducing the need for resection and with it the facial and functional impact. In recent years, the understanding of the biological behavior of maxillary injuries has undergone many changes. These changes in classification and nomenclature reveal that research continues on the characteristics of the injuries and the perspective of what was being managed as an ideal treatment. ${ }^{1}$ The World Health Organization reclassified the "keratocystic odontogenic tumor," which retakes its cyst category and is renamed "odontogenic keratocyst."

Between 1940 and 1970, maxillary lesions such as ameloblastomas were treated with enucleation because the options for performing the reconstructions were limited. Currently, there are new forms of reconstruction, so surgeons have taken surgical resection as the first choice in these patients with a wide margin of safety, based on the fact that it is the only treatment that guarantees a recurrence rate of $0 \%$. In the case of odontogenic keratocysts, the outlook is very similar; the reported recurrence rate after conservative management is between 11 and $54.5 \%$ depending on the technique. ${ }^{3,4}$ Updated literature emphasizes that the first-line treatment for odontogenic keratocyst is conservative; however, its high recurrence rate makes it considered not the ideal treatment plan. ${ }^{5}$ The treatment of these lesions should be aimed at improving the prognosis of the patient with the lowest potential for morbidity or recurrence of the lesion and this is achieved by understanding the diverse nature of the pathologies, their biological behavior and taking into account the age of the patients, the location, extent of the lesion and its histological characteristics. ${ }^{6,7}$

We consider that the enucleation and corticotomies technique with a rotary instrument with mucoperiosteal closure proposes a new alternative that, if it does not eliminate the risk of recurrence, allows for a decrease in the size of the lesion, facilitating resection and therefore achieving a favorable impact on function and aesthetics of patients. This conservative technique has been used in clinical practice to treat benign lesions of the jaws, including those with aggressive characteristics such as ameloblastomas.

One of the aspects that was taken into account when considering the surgical technique is the fact that both decompression and marsupialization, which are the most widely used procedures, require strict collaboration on the part of the patient since once performed the procedure the patient should be instructed to perform frequent flushing of the cavity by means of an implanted device until the desired result is evident. Unfortunately this patient collaboration is not always guaranteed; the cavity is usually filled with debris and becomes infected or plugged by increasing the pressure of the cyst with this material inside. The purpose of this study was to review the results of the proposed technique in benign lesions of the jaws over a period of 5 years and present it as an option for the management of these pathologies.

\section{MATERIALS AND METHODS}

A descriptive retrospective study of the patients treated in the Maxillofacial Surgery department of Clinica Universitaria Colombia from 2012 to 2016 was performed. The data collected included age of diagnosis, type of lesion, location of lesion, symptoms, radiographic characteristics, presence or absence of cortical expansion and perforation of the same, number and type of surgery, number of recurrences, time of follow-up and complications.

\section{DESCRIPTION OF THE TECHNIQUE}

ENUCLEATION, OSTEOTOMY, CURETTAGE, CORTICOTOMY WITH ROTARY INSTRUMENT AND MUCOPERIOSTIC CLOSURE

Under general anesthesia, an incision is made in the mucosa attached to the tumor trying to preserve the mucosa that is not adhered and a mucoperiosteal flap is elevated. Previously a planning in X-rays has been made to establish the margins of the lesion; the extraction of the teeth involved and a corticotomy are performed, which allow us to limit the healthy bone from the bone compromised by the lesion. Enucleation of 
the lesion is carried out carefully, in the case of keratocysts, preserving its capsule to continue with a curettage that allows reaching the areas with a difficult access. The cavity is prepared with abundant irrigation of saline with a peripheral osteotomy of all the walls of the residual cavity with a rotary instrument. As the recurrence rate of these lesions is directly related to the permanence of epithelial islands, this osteotomy has to be done carefully. During this process, irrigation is guaranteed to avoid damage by overheating of healthy bone or the roots of the teeth. The hermetic closure of the cavity is done in two layers, periosteum and mucosa. In addition to removing the entire epithelial component of the lesion, the residual cavity defect is used to contain the initial clot, so that bone neoformation with spontaneous regeneration is favored from it, without the need for grafts or any other reconstructive technique. Follow-up was initially every 8 days for one month and for the following 12 months each month with panoramic X-ray. In the second year the control was carried out every 6 months. The follow-up of the patients is very important, until 5 years of radiographic and clinical evidence of no signs or symptoms of locoregional recurrence, the definitive reconstructive procedures cannot begin.

\section{REPORT OF CASES}

\section{CASE 1}

A 26-year-old male patient referred for evaluation of mandibular lesion in July 2015. The finding of the lesion was incidental. The patient refers pain during chewing and intraoral inflammation. History included a third molar extraction 7 years prior and medical history of obsessive-compulsive disorder with fluoxetine intake. Clinically, there was no evidence of facial asymmetry; cavity in the right mandibular lingual region without the presence of secretions was observed. No dental mobility was evident.

Initial X-ray shows two corticalized radiolucent lesions in ramus and right mandibular body, the largest one in mandibular body, the smallest one related to first molar, apparently close to the mental and inferior alveolar dental nerve with inferior displacement of the same. First molar presented endodontic treatment (Fig 1). Enucleation, osteotomy, curettage and corticotomy with a rotary instrument were performed. The nerve was not involved in the surgical procedure. First molar was extracted. Biopsy proved keratocystic parakeratotic odontogenic tumor.

A 2-year post-op bone neoformation and changes in bone density of the right mandibular body were observed. The patient did not report symptoms, showed no signs of recurrence of the lesion, and no alterations in the sensitivity of the treated area (Fig 2).

\section{CASE 2}

A 15-year-old male patient referred for incidental findingofrightmandibularlesion(Fig 3). Anincisional biopsy was performed and a histopathological diagnosis of plexiform intraluminal unicystic ameloblastoma was established. It was decided to perform conservative surgical management with the proposed technique.

2 years after the surgical procedure, osseointegration implants were placed for aesthetic and functional requirements of antagonistic teeth. Four years later, osseointegrated implants are observed, no signs of osteolysis (Fig 4).

Currently, the patient is being followed up with panoramic X-ray; there are no signs of locoregional recurrence, no alterations in the sensitivity of the treated area.

\section{CASE 3}

A 11-year-old female patient who was referred from general dentistry for presenting an injury in the mandibular symphysis region confirmed with panoramic radiography. The patient consulted the dentist for presenting purulent exudate in lower anterior teeth. No medical or surgical history. Upon clinical examination, inflammation, erythema, and purulent exudate were evident in the mandibular anterior region (Fig 5).

The biopsy shows a keratocystic odontogenic tumor. The proposed technique is performed, and resolution of the lesion can be observed with adequate bone neoformation. Asymptomatic patient after 1 year of follow-up: lower anterior teeth separated from the cyst by an undamaged cortical bone, that is why their conservation is decided. Currently they are vital, without mobility, no alterations in the sensitivity of the treated area (Fig 6). 
ALDANAETAL
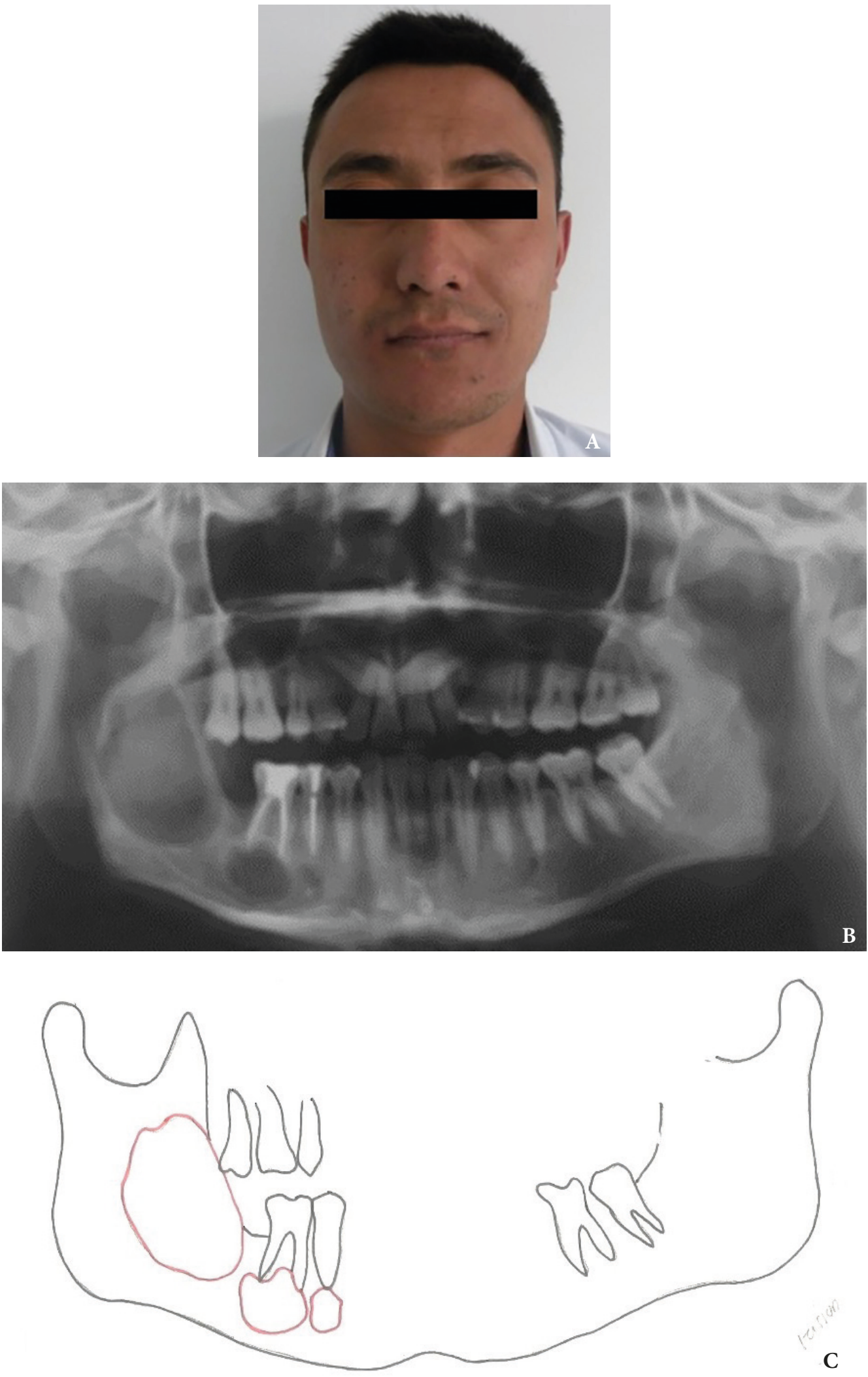

FIGURE 1. Case 1. A: Clinical picture. B: Initial X-ray shows two radiolucent corticalized lesions in the ramus and the right mandibular body: The larger one - in the mandibular ramus, the smaller one related to the first molar and apparent proximity to the inferior alveolar and mental nerve with inferior displacement of the last one. $\mathbf{C}$ : Lesions size scheme (red) for resection. 

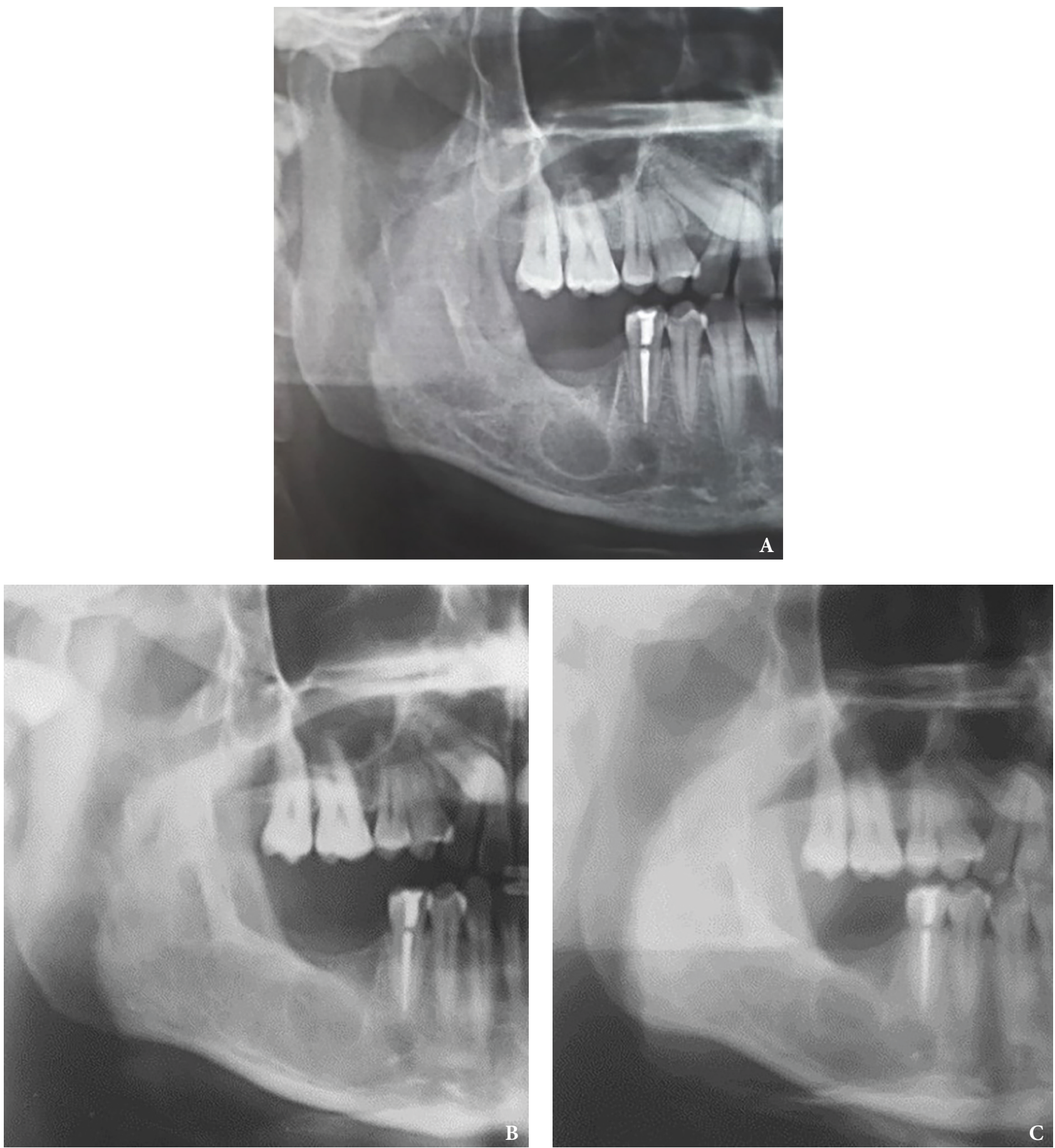

FIGURE 2. Case 1: Radiography. A: 6 months post-op. B: 1 year post-op. C: 2 years post-op. 

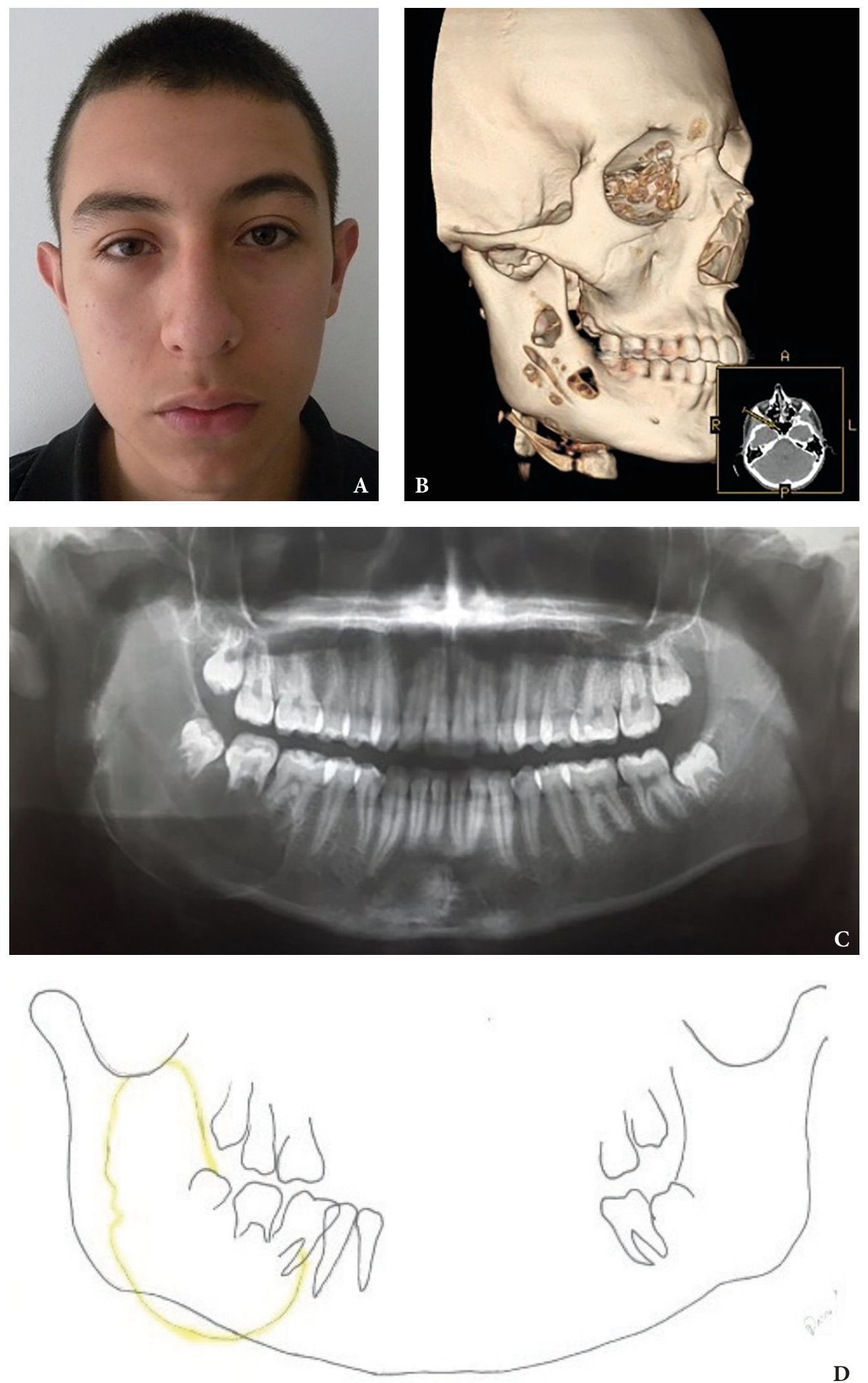

FIGURE 3. Case 2. A: Mild swelling in the right mandibular body, with tenderness. B: 3-dimensional computed tomography (CT) scan shows a lesion in the right mandibular body with perforation of the vestibular cortex. C: Panoramic X-ray shows a lesion in the right body and ramus related to first, second and third molar with apparent compromise of the inferior alveolar nerve. D: Lesion size scheme (yellow) for resection. 

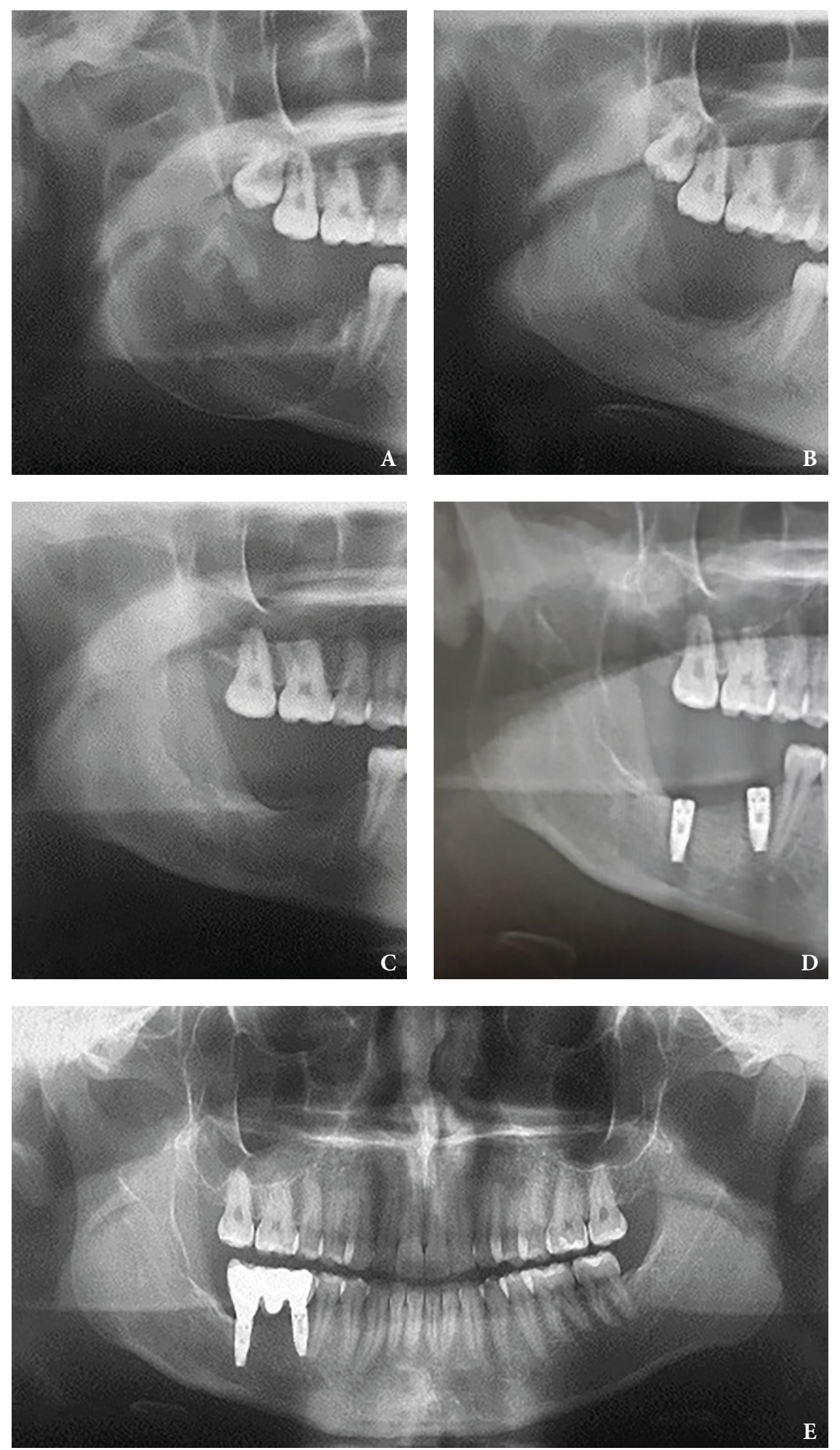

FIGURE 4. Case 2: Panoramic radiography. A: 3 months post-op. B: 6 months post-op. C: 1 year post-op. D: 2 years after the surgical procedure, osseointegration. The implants are placed for aesthetic and functional requirements of antagonistic teeth. E: 4 years post-op. 

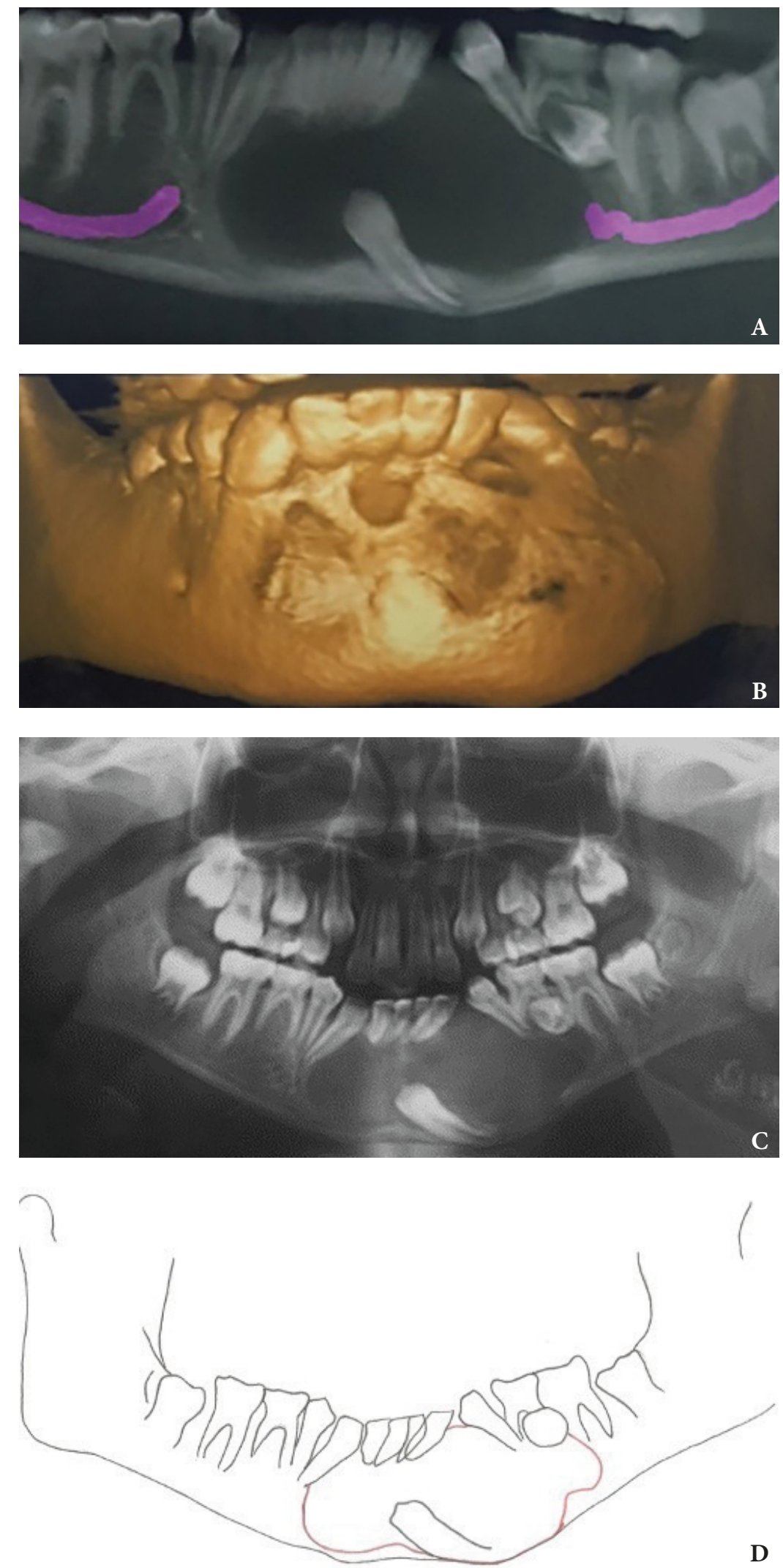

FIGURE 5. Case 3. CBCT $(\mathbf{A}, \mathbf{B})$ and panoramic radiography $(\mathbf{C})$ where extensive lesion is observed from the left first molar to the right second premolar in relation to the retained canine and premolar. $\mathbf{D}$ : Lesion size scheme (red) for resection. 

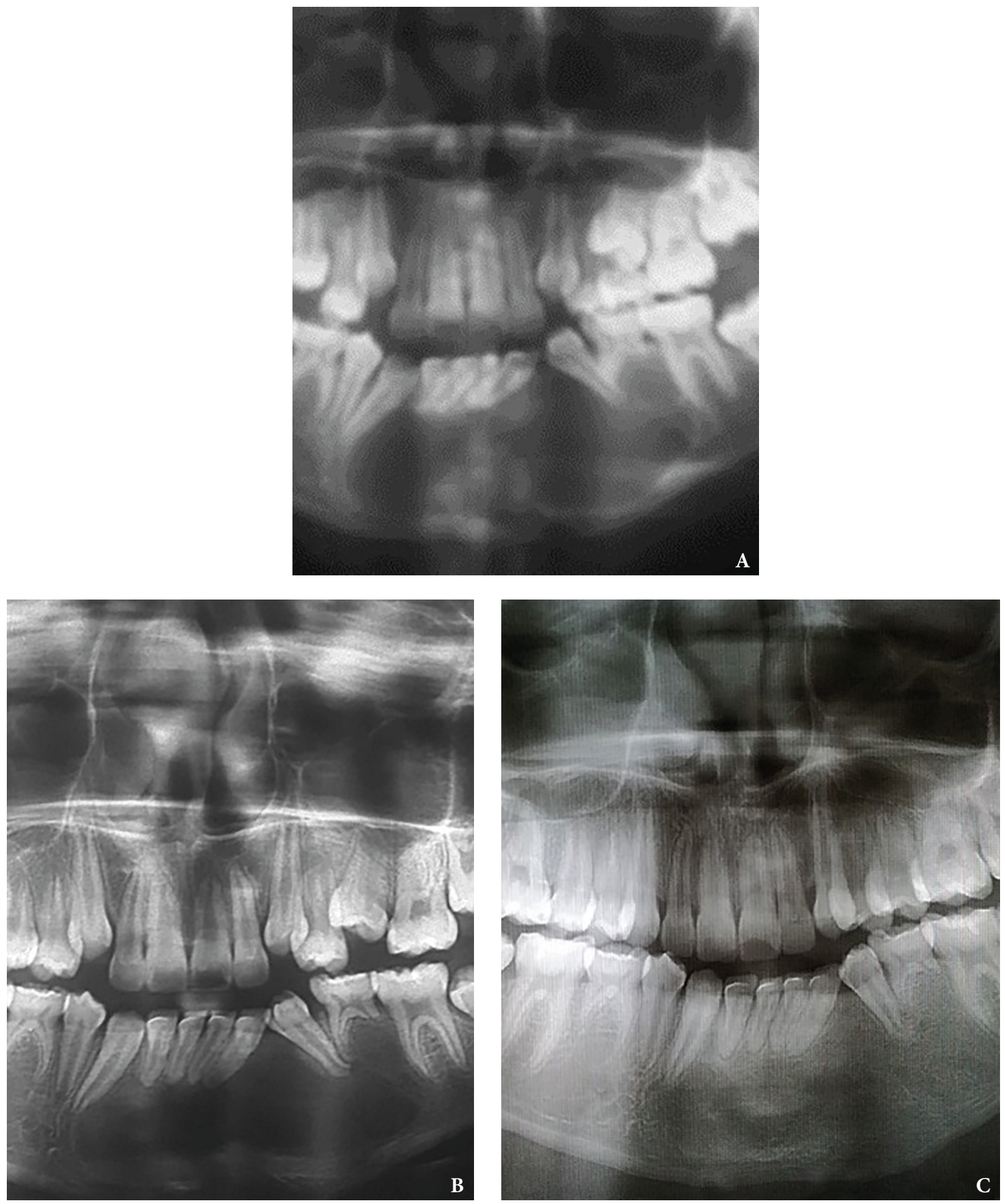

FIGURE 6. Case 3. A: Immediate post-op. B: 3 months post-op. C: 1 year post-op: Bone neoformation and changes in bone density are observed in the region of the mandibular symphysis. 


\section{CASE 4}

A 17-year-old female patient was referred from the general dentist for presenting a lesion in the mandibular symphysis region. A biopsy was performed with a histopathological diagnosis of keratocystic odontogenic tumor (Fig 7).

The same surgical technique was performed. Extraction of the included canine was done. 3 years later, bone neoformation can be observed, no alteration in the dental structures, no root resorption, no radiographic signs of recurrence of the lesion, no alterations in the sensitivity of the treated area. Currently asymptomatic (Fig 8).

\section{CASE 5}

A 49-year-old patient who consulted for rehabilitation with implants in the right mandibular area. The panoramic radiograph shows a multilocular lesion in the right mandibular body region associated with the included third molar, and an apparent relationship of the lesion with the inferior alveolar nerve is observed. Biopsy result reported keratocystic odontogenic tumor (Fig 9).

The second and third molars are removed and undergo the same surgical technique, without involving the inferior dental nerve. CT scan 3 years later shows an absence of injury, adequate corticalization and bone formation, continuity of the inferior alveolar nerve. Patient without alteration in sensitivity due to preservation of the inferio alveolar nerve during the surgical procedure (Fig 10).

\section{CASE 6}

A male patient diagnosed with keratocystic odontogenic tumor due to a history of enucleation without corticotomies and without adjuvant therapy 2 years prior to the consultation. He presented a multilocular lesion in the body and right mandibular ramus with apparent compromise of the inferior dental nerve and a second lesion in the region of the left mandibular angle (Fig 11).

Clinically, asymmetry was observed in the region of the right mandibular body, in the tomography no perforation of the cortical was observed. 4 months after the surgical procedure, bone neoformation and adequate healing of alveoli was already observed. This patient presented a recurrence of the right mandibular body lesion, underwent the same surgical technique with good results.
2 years after the procedure, a change in bone density was observed at the level of the body and the right mandibular ramus and the left mandibular angle. Asymptomatic patient, no alterations in the sensitivity of the treated area, awaiting rehabilitation with implants. Due to the possibility of recurrence of the lesion, a minimum of 5 years of follow-up was proposed to start rehabilitation with implants (Fig 12).

\section{CASE 7}

A male patient treated with the same technique with evidence in panoramic radiography of resolution of the lesion 2 years after the surgical procedure. Currently he is asymptomatic (Figs 13 and 14).

\section{DISCUSSION}

Odontogenic keratocyst was first described by that name in 1956 and has long been associated with a tendency to recur. Currently, the odontogenic keratocyst was defined by the World Health Organization as an intraosseous lesion characterized by the presence of a thin and regular layer of keratinized stratified squamous epithelium and the presence of hyperchromatic basal cells. It corresponds to 10 to $20 \%$ of odontogenic cysts and is the third most common cyst of the jaw cysts. Odontogenic keratocyst has a slight predilection for men and a peak incidence that is reported in the second and third decades of life. ${ }^{2,8}$ Upon $80 \%$ odontogenic keratocysts are located in the jaw with a predilection for the body and the mandibular ramus. When they occur in the maxilla, they are located in the retromolar region and tuberosity and are related to Nevoid Basal Cell Carcinoma syndrome., ${ }^{9,10}$

Ameloblastoma, on the other hand, has been defined as a benign intraosseous tumor. A progressive growing odontogenic epithelial neoplasm characterized by generating expansion and a tendency to local recurrence if it is not adequately eliminated. Although it is a rare tumor and corresponds to $1 \%$ of maxillary tumors, ameloblastoma is the most common tumor within the odontogenic tumor family and its maximum incidence peak occurs in the fourth and fifth decades of life without sex predilection. Its most frequent location is the mandible in its posterior region, followed by the mandibular anterior region, the maxillary posterior region and the maxillary anterior region. ${ }^{2,11}$ 

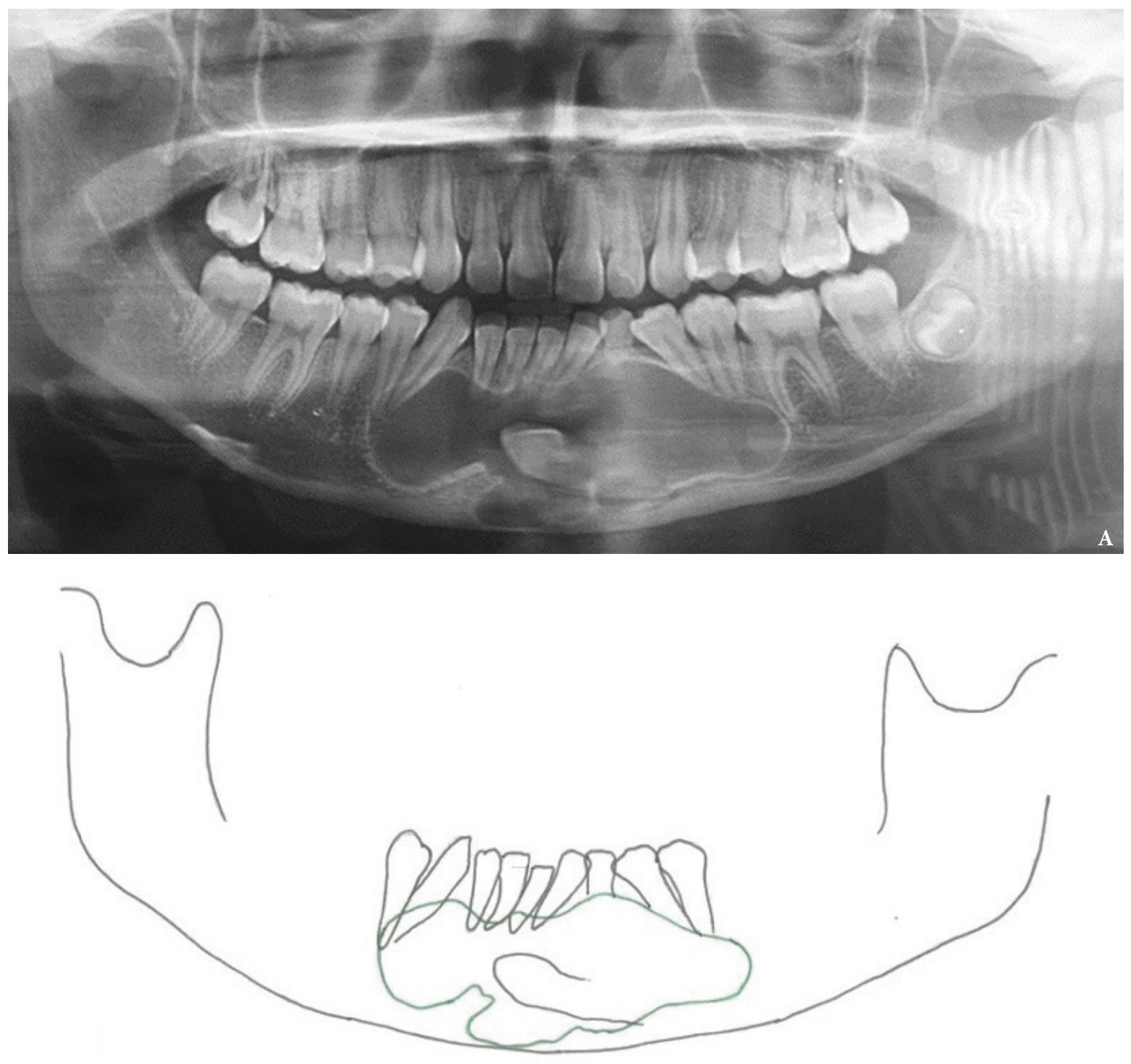

FIGURE 7. Case 4. A: Initial X-ray. B: Lesion size scheme (green) for resection. 

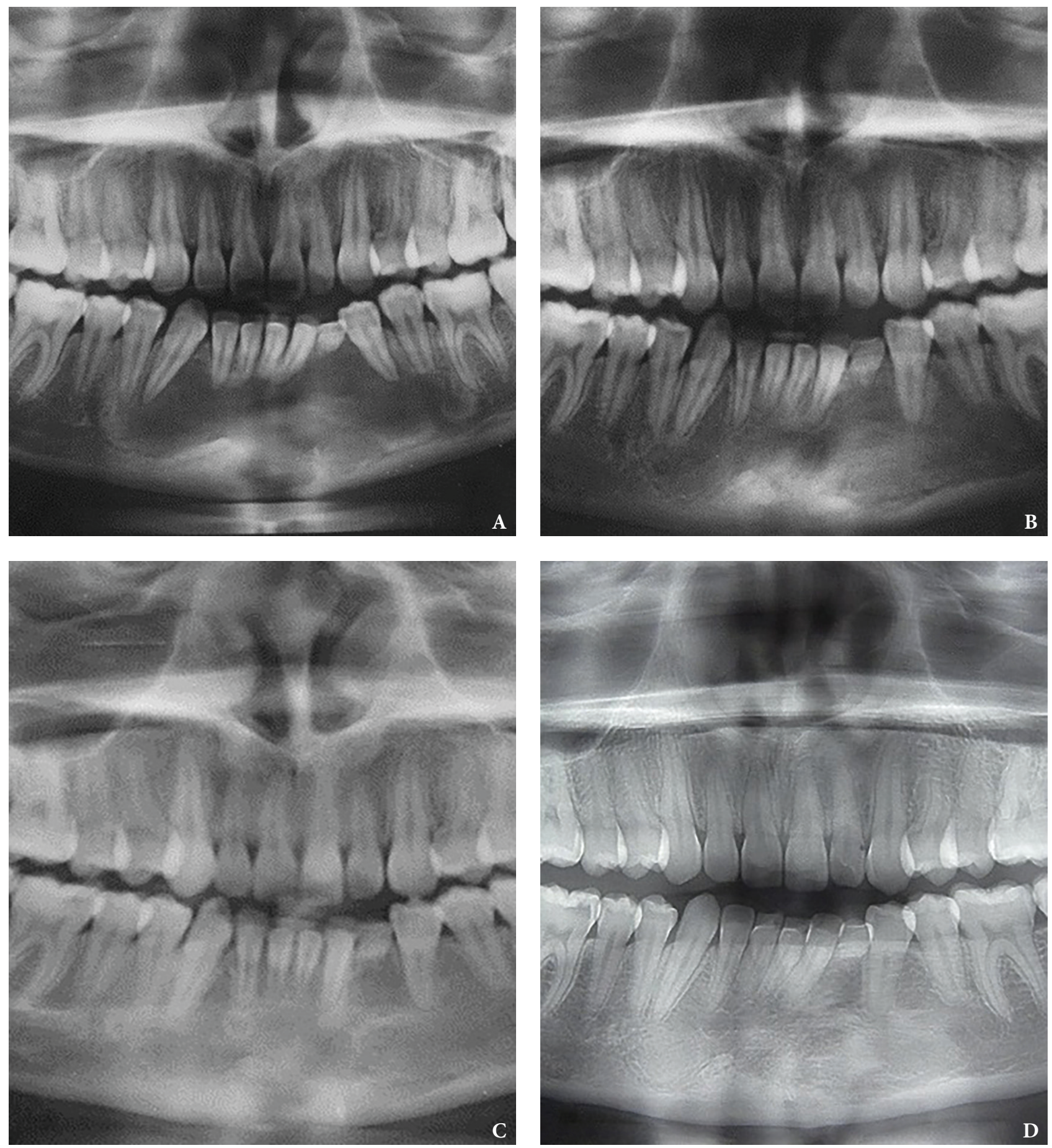

FIGURE 8. Case 4: Cropped panoramic radiography. A: Immediate post-op. B: 1 year post-op. C: 2 years post-op. D: 3 years post-op. 

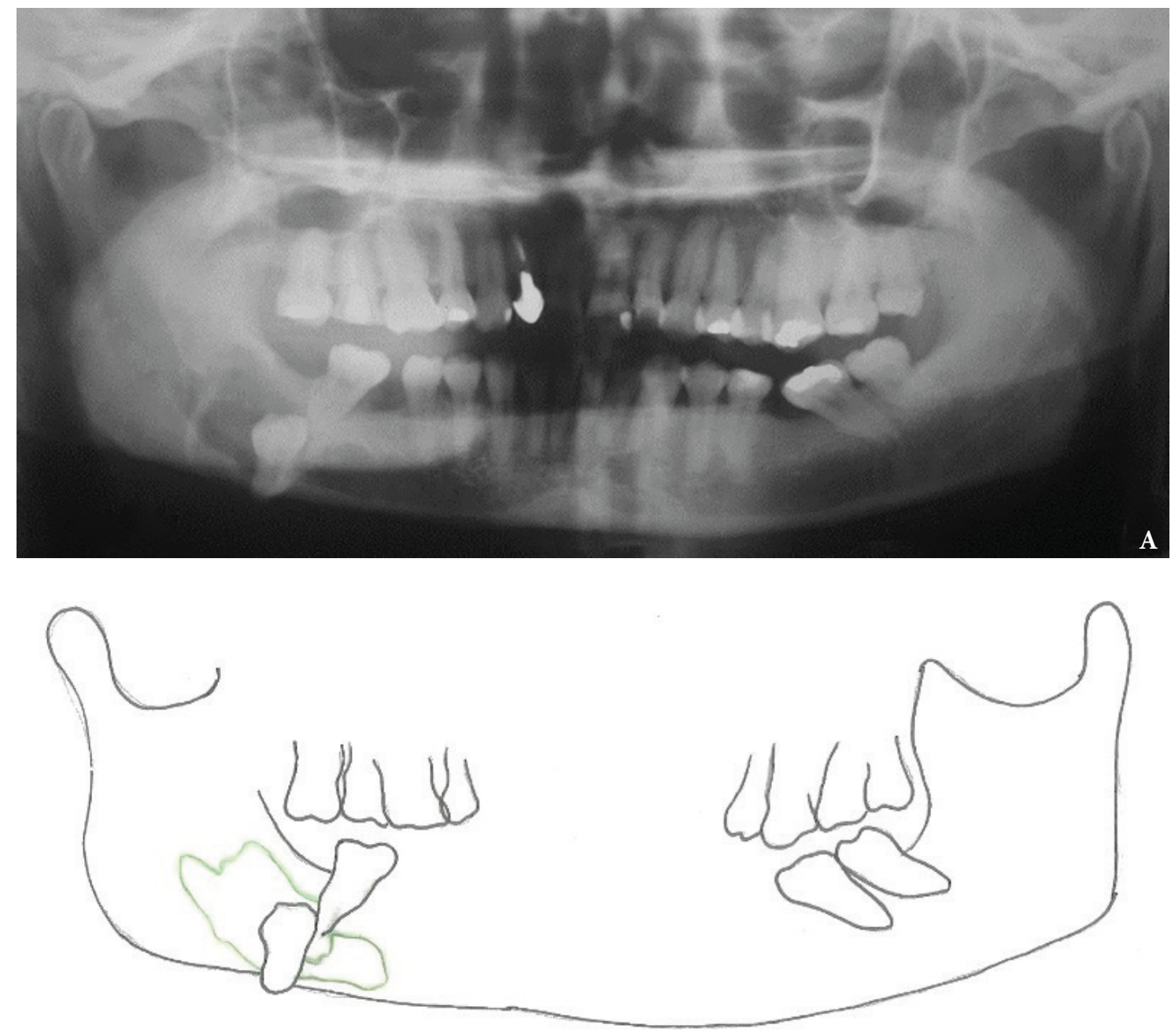

FIGURE 9. Case 5. A: Initial X-ray. B: Lesion size scheme (green) for resection. 

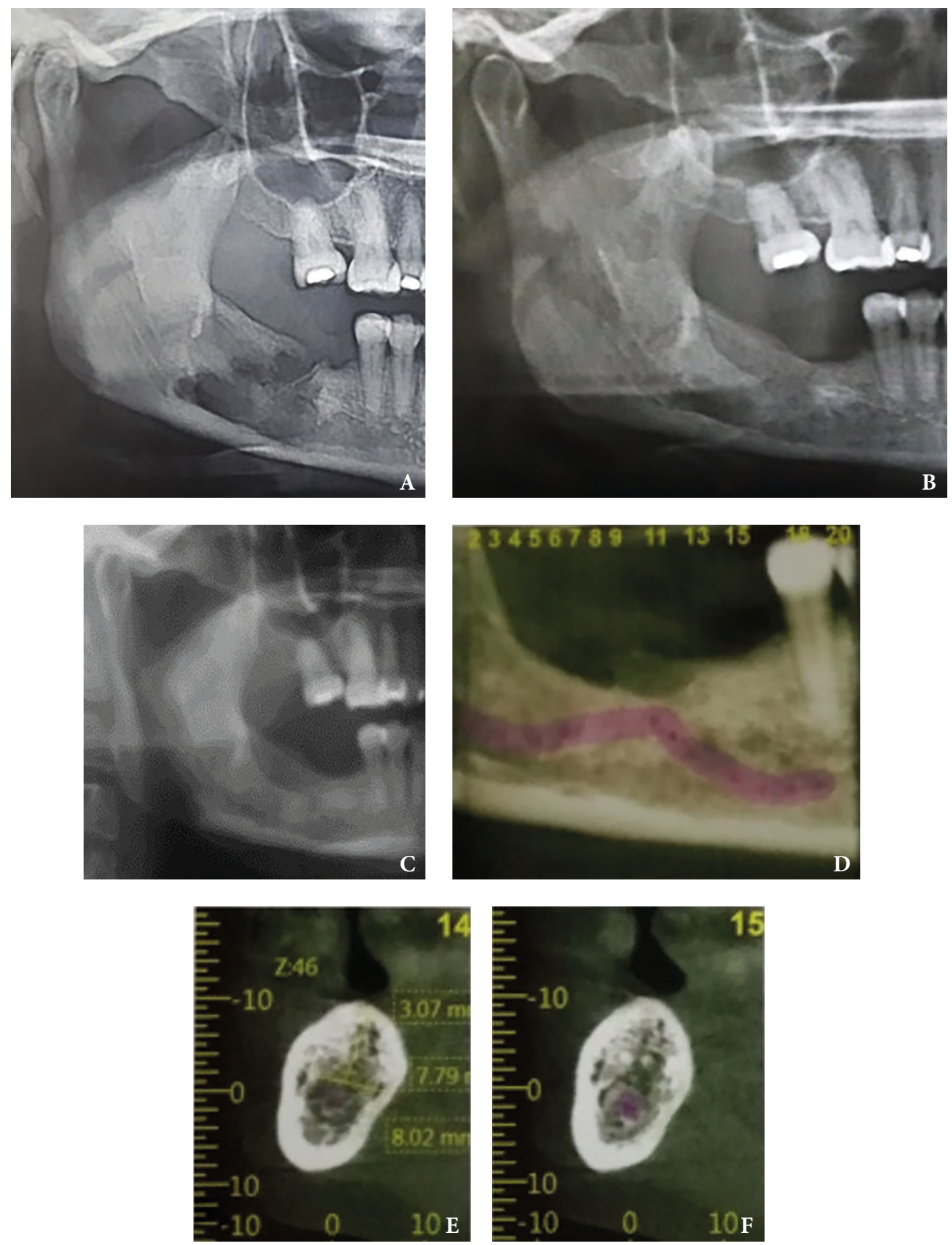

FIGURE 10. Case 5. A: 3 months post-op. B: 6 months post-op. C: 1 year post-op. D-F: CT scans 3 years later show absence of injury, adequate corticalization and bone formation, continuity of the inferior alveolar nerve. 

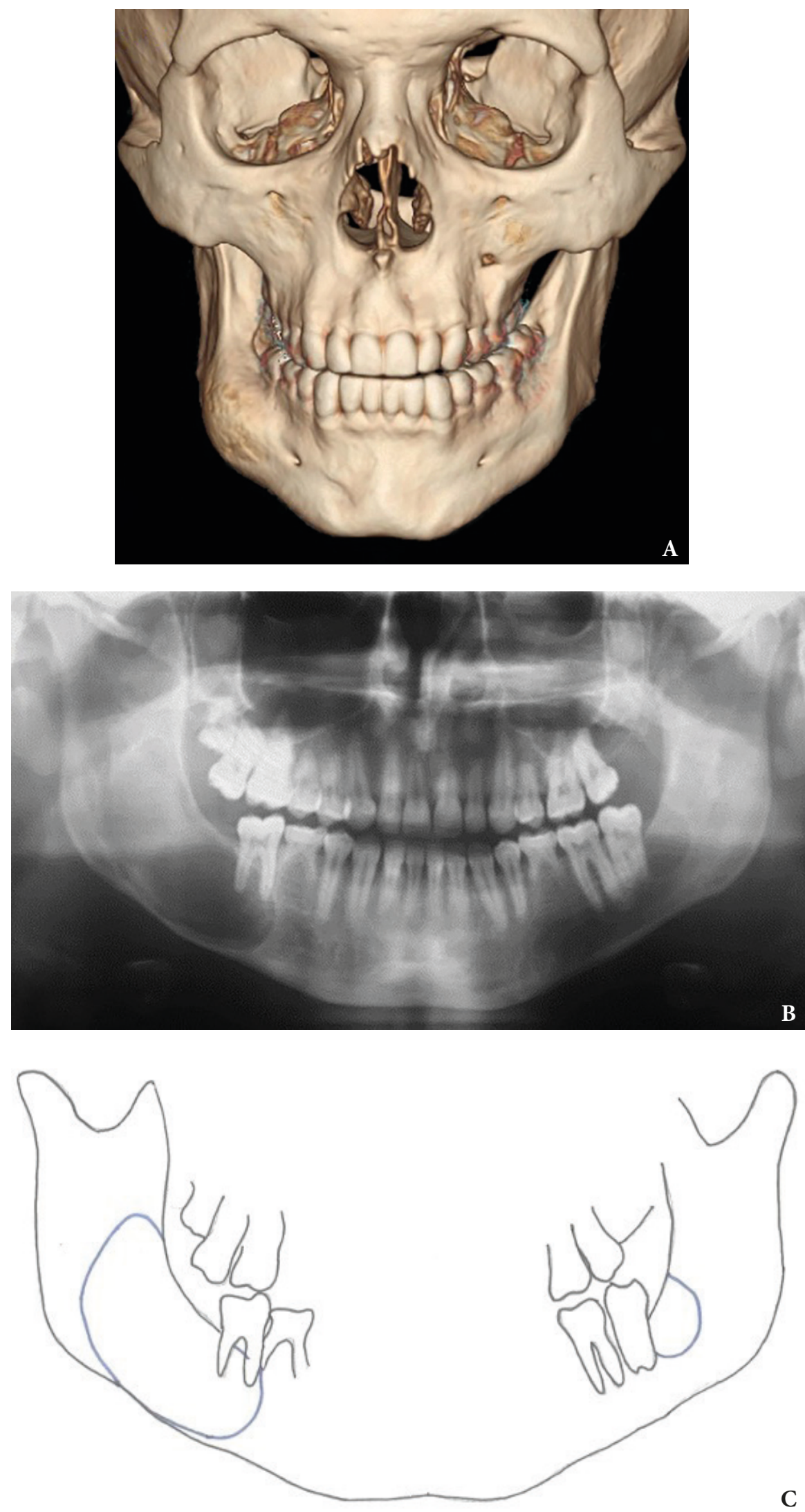

FIGURE 11. Case 6. A: 3-dimensional CT scan showing buccal cortex expansion without erosion. B: Initial X-ray. C: Lesions ` size scheme (blue) for resection. 

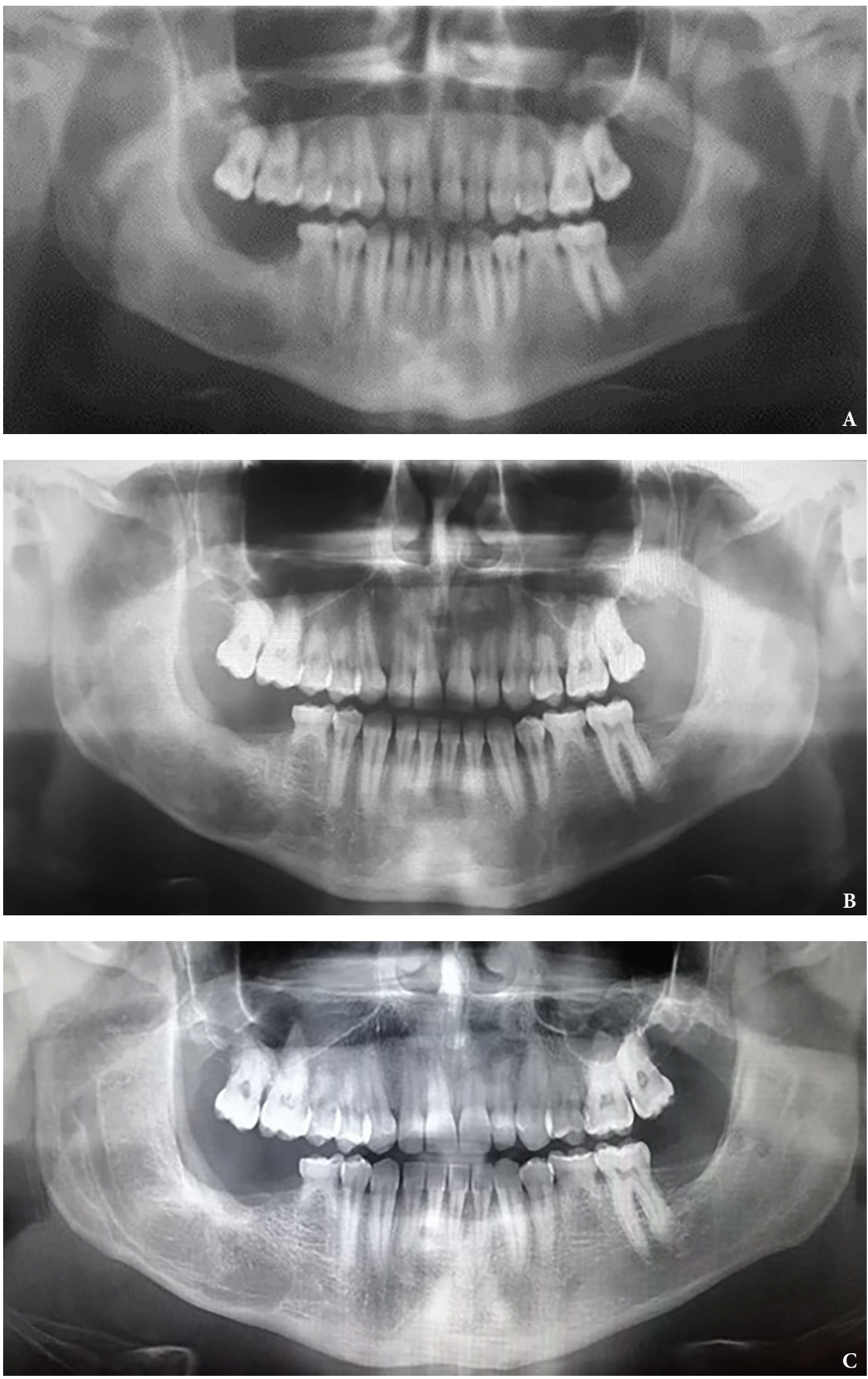

FIGURE 12. Case 6: Panoramic radiography. A: 7 months post-op. B: 1 year post-op. C: 2 years post-op. 

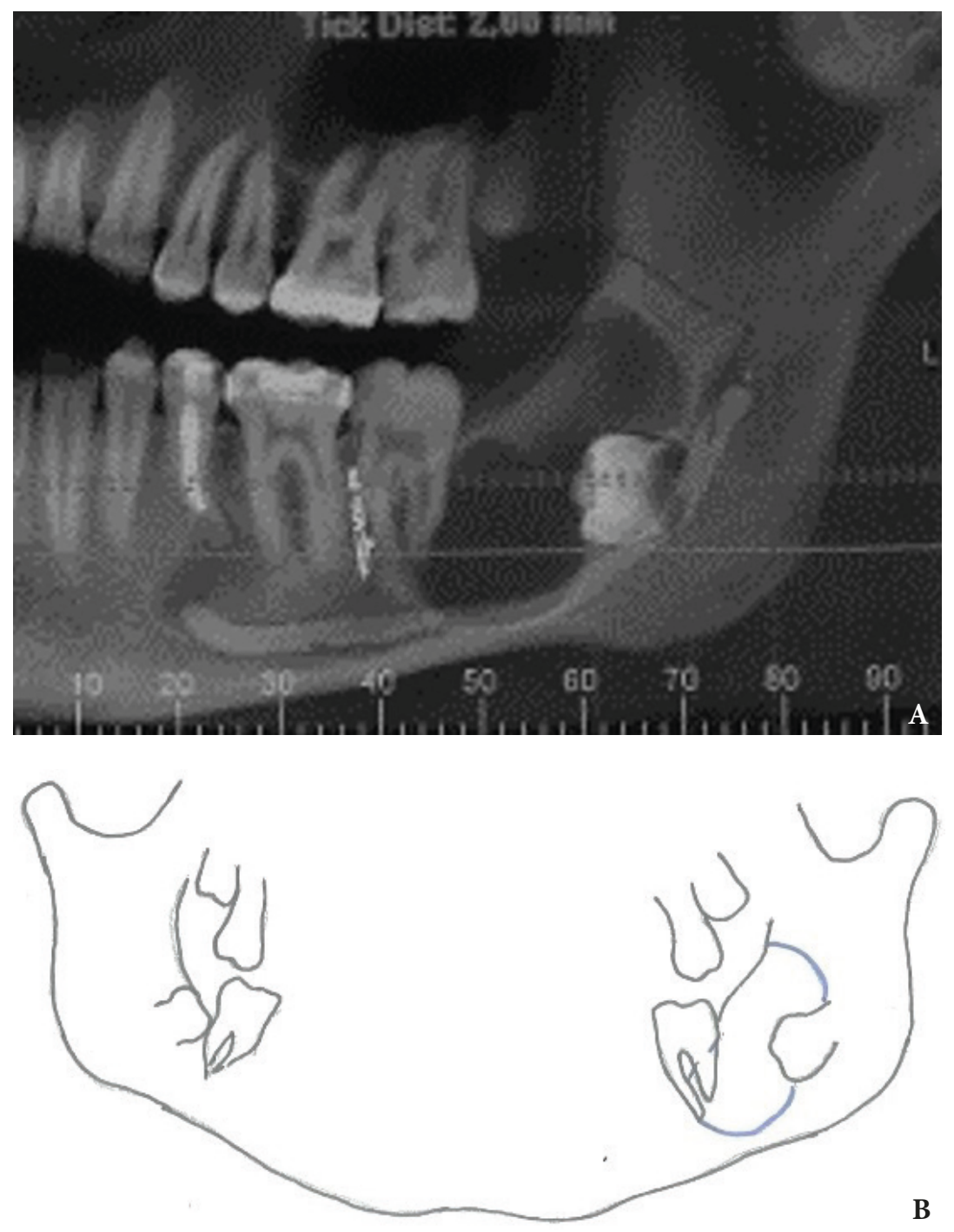

FIGURE 13. Case 7. A: Initial X-ray. B: Lesion size scheme (blue) for resection. 

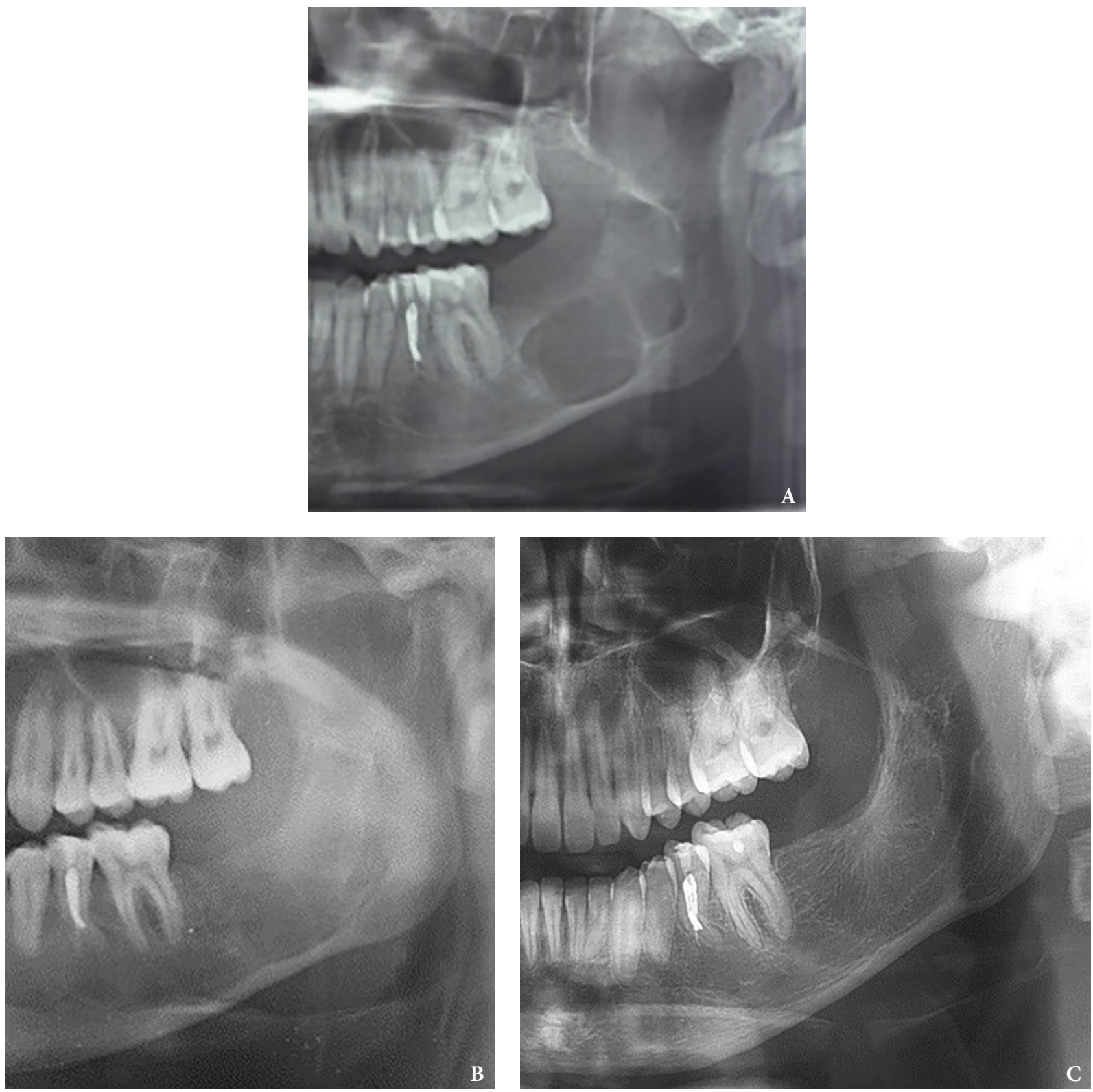

FIGURE 14. Case 7. A: 2 months post-op. B: 6 months post-op. C: 2 years post-op. 
All the surgical techniques for the management of these lesions have a common objective that consists of eradicating the cyst and reducing the risk of recurrence and surgical morbidity. Over time, discussions have been held on the most appropriate form of clinical management of these lesions and their treatment has been controversial and variable. It has been proposed from conservative management to surgical resection, giving importance to the complete removal of the epithelial component of the cyst, which is the only thing that guarantees non-recurrence of the lesion. ${ }^{3,9}$

Marsupialization and decompression have now been accepted as conservative treatment modalities for many cysts and lesions of the jaws, including dentigerous cysts, root cysts, keratocysts, and cystic ameloblastomas. ${ }^{12,13}$

Just as the literature accepts them as the firstchoice treatment, there are authors who describe that they are effective but followed by a secondary treatment such as enucleation, curettage and bone resection. ${ }^{14,15}$ The surgical resection technique has remained unchanged since its origins. It implies the elimination of the lesion with margins of 1 $\mathrm{cm}$. Lesions of the lower jaw have an additional component and that is the relationship of the lesion to the lower dental nerve. ${ }^{16}$ In cases of benign lesions for both keratocysts and ameloblastomas, there is still controversy. Some authors consider that in large lesions it must be sacrificed. Specifically in follicular ameloblastoma, it is essential to eliminate it since perineural invasion has been observed in specimens studied histopathologically. ${ }^{12,17}$ None of the patients who underwent the proposed technique presented an alteration in the sensitivity of the affected area. On radiographs and tomography, it was observed that the lesions displaced the inferior dental nerve and were close to the mental nerve. The technique was performed without compromising the vascular component since they were benign lesions and, to date, no alteration in sensitivity has been reported, and this procedure was not related to an increase in the recurrence rate. Pogrel is one of the authors who have most investigated techniques for managing keratocysts. In 2004 he proposed a new technique that combines enucleation of the lesion with peripheral osteotomies to remove remaining cysts that could remain in the adjacent bone. ${ }^{6} \mathrm{He}$ combined the technique with the placement of drainage devices that prevent pressure increase. He observed that over time the cyst epithelium became thinner and easier to enucleate and that a new epithelium very similar to the normal epithelium was formed. With this decompression, enucleation and peripheral osteotomy technique in 29 patients, with follow-up for a minimum period of 4 years and a maximum of 5, no recurrence was observed. ${ }^{18,19}$

Nakamura et $\mathrm{a}^{20}$ publish in their article that of 23 lesions managed with marsupialization, enucleation and curettage, 5 disappeared completely and did not require resection and 6 presented recurrence in a variable time from 1 to 16 years. In this study, recurrences were observed in all the maxillary sites, but a predilection was observed in the angle region and the mandibular ramus. No statistically significant difference was observed in unilocular or multilocular lesions with a respective percentage of $21.4 \%$ versus $20 \% .{ }^{20}$ According to what was published in the article by Cunha et $\mathrm{al}^{21}$, if the tooth involved in the injury is not extracted, there is a $67 \%$ chance of recurrence of the injury, while if the tooth is extracted, there is a $100 \%$ guarantee that recurrence will not occur. This phenomenon is explained by the difficulty that exists for the complete removal of the epithelium from the roots of the teeth, the periodontal ligament and the furcation area. ${ }^{21}$ Anavi et $\mathrm{al}^{13}$ reported that the mean reduction after decompression was $78.9 \%$ with an average decompression time of 9.2 months. For young patients, they indicate that the decompression time is shorter with an average of 7.6 months explained by the high osteogenic activity of children. ${ }^{13}$

Two years post-op, patient of case 6 showed recurrence of the lesion in the right mandibular body. A second surgery was performed in which the same technique was used. Two years of post-op of the second surgery, the patient did not required new interventions and is awaiting rehabilitation with implants. So far, it is not possible to emphasize whether there are differences in the success of the treatment in unilocular or multilocular lesions or if it depends on the histological type, since all cases were offered the same opportunity with the same technique based on the principle of reducing the sequelae and morbidity with conservative management with excellent results. 


\section{CONCLUSIONS}

Conservativemanagementhasbeen underestimated; the literature is not benevolent with this management plan. The proposed technique has been shown to have a high success rate, which, although it does not eliminate the risk of recurrence, has a considerable impact on the patient by reducing the need for resection and with this facial and functional damage.

Patients with recurrence of the lesion after conservative management can undergo the same procedure with high success rates since the lesions are smaller and facilitate enucleation. The monitoring of these patients is part of their treatment and must be observed both clinically and radiographically for at least 10 years.

\section{FUNDINGS}

No funding was received for this study.

\section{CONFLICT OF INTERESTS}

The authors declare no conflict of interest.

\section{PATIENT CONSENT}

Patients provided written consent of the use of the images.

\section{REFERENCES}

1. Keiser G. Odontogenic cysts and tumors of the maxilla: controversies in surgical management. Oper Tech Otolaryngol Head Neck Surg 1999;10(2):140-7.

2. El-Naggar AK, Chan JKC, Grandis JR, Takata T, Slootweg PJ. World Health Organization classification of head and neck tumours. 4th ed. Lyon: IARC; 2017.

3. Bharani S, Lakshmi S, Kamath R, Hammannavar R. Keratocystic odontogenic tumor: case report, treatment review and future diagnostic trends. J Oral Maxillofac Surg Med Pathol 2013;25(1):85-92.

4. Haq J, Siddiqui S, McGurk M. Argument for the conservative management of mandibular ameloblastomas. $\mathrm{Br} \quad \mathrm{J}$ Oral Maxillofac Surg 2016;54(9):1001-5.

5. Simiyu BN, Butt F, Dimba EA, Wagaiyu EG, Awange DO, Guthua SW, Slootweg PJ. Keratocystic odontogenic tumours of the jaws and associated pathologies: a 10-year clinicopathologic audit in a referral teaching hospital in Kenya. J Craniomaxillofac Surg 2013;41(3):230-4.
6. Pogrel MA, Jordan RKC. Marsupialization as a definitive treatment for the odontogenic keratocyst. $J$ Oral Maxillofac Surg 2004;62(6):651-5.

7. Leung YY, Lau SL, Tsoi KY, Ma HL, Ng CL. Results of the treatment of keratocystic odontogenic tumours using enucleation and treatment of the residual bony defect with Carnoy's solution. Int J Oral Maxillofac Surg 2016;45(9):1154-8.

8. Arce K, Streff CS, Ettinger KS. Pediatric odontogenic cysts of the jaws. Oral Maxillofac Surg Clin North Am 2016;28(1):21-30.

9. Pitak-Arnnop P, Chaine A, Oprean N, Dhanuthai K, Bertrand JC, Bertolus C. Management of odontogenic keratocysts of the jaws: a ten-year experience with 120 consecutive lesions. J Craniomaxillofac Surg 2010;38(5):358-64.

10. Figueroa A, Correnti M, Avila M, Andea A, DeVilliers P, Rivera H .Keratocystic odontogenic tumor associated with nevoid basal cell carcinoma syndrome: similar behavior to sporadic type? Otolaryngology Head Neck Surg 2010;142(2):179-83.

11. MacDonald D. Lesions of the jaws presenting as radiolucencies on cone-beam CT. Clin Radiol 2016;71(10):972-85.

12. Abrahams JM, McClure SA. Pediatric odontogenic tumors. Oral Maxillofac Surg Clin North Am 2016;28(1):45-58.

13. Anavi Y, Gal G, Miron H, Calderon S, Allon DM. Decompression of odontogenic cystic lesions: clinical long-term study of 73 cases. Oral Surg Oral Med Oral Pathol Oral Radiol Endod 2011;112(2):164-9.

14. Johnson NR, Batstone MD, Savage NW. Management and recurrence of keratocystic odontogenic tumor: a systematic review. Oral Surg Oral Med Oral Pathol Oral Radiol 2013;116(4):e271-e276.

15. Hou R, Zhou H. Articles of marsupialization and decompression on cystic lesions of the jaws: a literature review. J Oral Maxillofac Surg Med Pathol 2013;25(4):299-304.

16. Morgan TA, Burton CC, Qian F. A retrospective review of treatment of the odontogenic keratocyst. $J$ Oral Maxillofac Surg 2005;63(5):635-9.

17. Payne S, Lighthall J, Albert T. Management of ameloblastoma in the pediatric population. Oper Tech Otolaryngol Head Neck Surg 2015;26(3):168-74.

18. Pogrel MA. The keratocystic odontogenic tumor. Oral Maxillofac Surg Clin North Am 2013;25(1):21-30.

19. Pogrel MA. The keratocystic odontogenic tumour (KCOT)--an odyssey. Int J Oral Maxillofac Surg 2015;44(12):1565-8.

20. Nakamura N, Mitsuyasu T, Mitsuyasu Y, Taketomi T, Higuchi Y, Ohishi M. Marsupialization for odontogenic keratocysts: long-term follow-up analysis of the effects and changes in growth characteristics. Oral Surg Oral Med Oral Pathol Oral 
Radiol Endod 2002;94(5):543-53.

21. Cunha JF, Gomes CC, de Mesquita RA, Andrade Goulart EM, de Castro WH, Gomez RS. Clinicopathologic features associated with recurrence of the odontogenic keratocyst: a cohort retrospective analysis. Oral Surg Oral Med Oral Pathol Oral Radiol 2016;121(6):629-35. 